\title{
A NOTE ON POINT SOURCE DIFFRACTION BY A WEDGE
}

\author{
A. D. RAWLINS
}

Received 18 June 2003

The object of this paper is to give new expressions for the wave field produced when a time harmonic point source is diffracted by a wedge with Dirichlet or Neumann boundary conditions on its faces. The representation of the total field is expressed in terms of quadratures of elementary functions, rather than Bessel functions, which is usual in the literature. An analogous expression is given for the three-dimensional free-space Green's function.

\section{Introduction}

The wave field produced when a line or point source is diffracted by an ideal wedge was first given by Macdonald [5] following Poincaré [7] in terms of a Fourier-Bessel series some time ago. Since that time, no substantial simplification has been carried out - see Bowman et al. [1]. By using this Fourier-Bessel series, it is possible by means of an integral representation given by Lamb [4] and various results due to Macdonald [6] to produce a new representation that avoids Bessel functions completely and only involves elementary trigonometric expressions. To the author's knowledge, these results are new, although it is surprising that such results have not appeared before since the approach is quite elementary. The new representation offers a more compact representation for the total wave field that is easy to compute. A new representation is also given for a harmonic point source, that is, the three-dimensional free-space Green's function for the reduced wave equation.

\section{Formulation of the boundary value problem}

We will assume a harmonic point wave source $u_{0} e^{-i w t}$, where $w$ is the angular frequency located at cylindrical polar coordinates $Q\left(r_{0}, \theta_{0}, z_{0}\right)$, where the field at any other point $P(r, \theta, z)$ is given by

$$
u_{0}(r, \theta, z)=\frac{e^{i k R}}{R}
$$




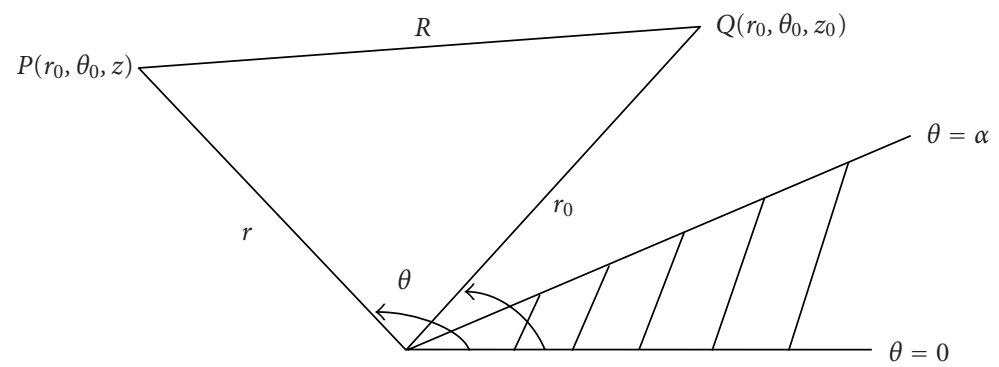

Figure 2.1. The $z$-axis lies along the edge of the wedge. The radial distances $r$ and $r_{0}$ lie in a plane perpendicular to the $z$-axis. The distance $R=\left\{r^{2}+r_{0}^{2}-2 r r_{0} \cos \left(\theta-\theta_{0}\right)+\left(z-z_{0}\right)^{2}\right\}^{1 / 2}$ lies between the point $P$ in the plane $z=z$ and $Q$ in the plane $z=z_{0}$.

where $k=2 \pi / \lambda\left(\lambda\right.$ is the wave length) and $R=\left\{r^{2}+r_{0}^{2}-2 r r_{0} \cos \left(\theta-\theta_{0}\right)+\left(z-z_{0}\right)^{2}\right\}^{1 / 2}$ is the distance between the source point $Q$ and any other point $P$. A solid wedge is assumed to occupy the region $(r \geq 0,0 \leq \theta \leq \alpha,-\infty<z<\infty)$ and the source point lies in the complementary region, thus $0<r_{0}, \alpha<\theta_{0}<2 \pi$, and $-\infty<z_{0}<\infty$; see Figure 2.1.

The unique solution $u^{D}$ of the Dirichlet $(u=0$ on $\theta=0, \theta=\alpha)$ and $u^{N}$ of the Neu$\operatorname{mann}(\partial u / \partial \theta=0$ on $\theta=0, \theta=\alpha)$ boundary value problem that satisfies the reduced wave equation, the radiation, and edge condition, is well known, and is given by Bowman et al. $[1]$ as

$$
u^{D / N}=\frac{2 \pi}{\alpha} \sum_{n=0}^{\infty} \in_{n} \sin _{\cos }\left\{v_{n} \theta_{0}\right\}_{\cos }^{\sin }\left\{v_{n} \theta\right\} I_{n}\left(r, z ; r_{0} z_{0}\right)
$$

where

$$
I_{n}\left(r, z ; r_{0}, z_{0}\right)=\int_{-\infty}^{\infty} J_{v_{n}}\left(h r_{<}\right) H_{v_{n}}^{(1)}\left(h r_{>}\right) e^{-\left|z-z_{0}\right| \sqrt{\left(h^{2}-k^{2}\right)}} \frac{h d h}{\sqrt{\left(h^{2}-k^{2}\right)}}
$$

and $r_{>}=r_{0}, r_{<}=r$ when $r_{0}>r$; and $r_{>}=r, r_{<}=r_{0}$ when $r>r_{0}$, with $v_{n}=n \pi / \alpha, \in_{0}=1 / 2$, and $\in_{n}=1, n \geq 1$; and the square root is defined by

$$
\begin{aligned}
\sqrt{\left(h^{2}-k^{2}\right)} & =\sqrt{\left|h^{2}-k^{2}\right|}, \quad h^{2}>k^{2}, \\
& =-i \sqrt{\left|k^{2}-h^{2}\right|}, \quad h^{2}<k^{2} .
\end{aligned}
$$

Although Bowman et al. [1] state that $I_{n}$ cannot be simplified, we show that it can be expressed in terms of elementary functions as follows.

We can write (2.3) as

$$
\begin{aligned}
I_{n}= & \int_{0}^{\infty} J_{v_{n}}\left(h r_{<}\right) H_{v_{n}}^{(1)}\left(h r_{>}\right) e^{-\left|z-z_{0}\right| \sqrt{\left(h^{2}-k^{2}\right)}} \frac{h d h}{\sqrt{\left(h^{2}-k^{2}\right)}} \\
& -\int_{0}^{\infty} J_{v_{n}}\left(-h r_{<}\right) H_{v_{n}}^{(1)}\left(-h r_{>}\right) e^{-\left|z-z_{0}\right| \sqrt{\left(h^{2}-k^{2}\right)}} \frac{h d h}{\sqrt{\left(h^{2}-k^{2}\right)}} .
\end{aligned}
$$


And by using the fact that $H_{v}^{(1)}\left(z e^{i \pi}\right)=-e^{-i v \pi} H_{v}^{(2)}(z)$ and $J_{v}\left(z e^{i \pi}\right)=e^{v \pi i} J_{v}(z)$, we get

$$
\begin{aligned}
I_{n} & =\int_{0}^{\infty} J_{v_{n}}\left(h r_{<}\right)=\left\{H_{v_{n}}^{(1)}\left(h r_{>}\right)+H_{v_{n}}^{(2)}\left(h r_{<}\right)\right\} e^{-\left|z-z_{0}\right| \sqrt{\left(h^{2}-k^{2}\right)}} \frac{h d h}{\sqrt{\left(h^{2}-k^{2}\right)}} \\
& =2 \int_{0}^{\infty} J_{v_{n}}\left(h r_{<}\right) J_{v_{n}}\left(h r_{>}\right) e^{-\left|z-z_{0}\right| \sqrt{\left(h^{2}-k^{2}\right)}} \frac{h d h}{\sqrt{\left(h^{2}-k^{2}\right)}} .
\end{aligned}
$$

But Lamb [4] has shown that for the root determination (2.4),

$$
\frac{e^{-\left|z-z_{0}\right| \sqrt{\left(h^{2}-k^{2}\right)}}}{\sqrt{\left(h^{2}-k^{2}\right)}}=\int_{0}^{\infty} J_{0}(h t) \frac{e^{i k \sqrt{\left(t^{2}+\left|z-z_{0}\right|^{2}\right)}}}{\sqrt{\left(t^{2}+\left|z-z_{0}\right|^{2}\right)}} t d t .
$$

Substituting (2.7) into (2.6) and interchanging the order of integration (permissible since the double integral is uniformly convergent before and after the interchange of order of integration), we get

$$
I_{n}=2 \int_{0}^{\infty} \frac{t e^{i k \sqrt{\left(t^{2}+\left|z-z_{0}\right|^{2}\right)}}}{\sqrt{\left(t^{2}+\left|z-z_{0}\right|^{2}\right)}}\left(\int_{0}^{\infty} h J_{v_{n}}\left(h r_{0}\right) J_{v_{n}}(h r) J_{0}(h t) d h\right) d t
$$

The integral in the brackets of the last expression has been evaluated by Macdonald [6] as

$$
\begin{aligned}
W & =\int_{0}^{\infty} h J_{v_{n}}\left(h r_{0}\right) J_{v_{n}}(h r) J_{0}(h t) d h \\
& =i \sqrt{\frac{2}{\pi}} \frac{\sin v_{n} \pi}{r r_{0} \pi} Q_{v_{n}-1 / 2}^{1 / 2}(\mu)(\mu)\left(\mu^{2}-1\right)^{-1 / 4}, \quad|t|>\left|r+r_{0}\right|, \mu>1 \\
& =\frac{1}{\sqrt{2 \pi}} \frac{1}{r r_{0}}\left(1-\mu^{2}\right)^{-1 / 4} P_{v_{n-1 / 2}}^{1 / 2}(-\mu), \quad\left|r+r_{0}\right|>|t|>\left|r-r_{0}\right|,-1<\mu<1 \\
& =0, \quad\left|r-r_{0}\right|>|t|,
\end{aligned}
$$

where $-\mu=\left(r^{2}+r_{0}^{2}-t^{2}\right) / 2 r r_{0}$.

But the associated Legendre functions $P_{v_{n}-1 / 2}^{1 / 2}(-\mu)$ and $Q_{v_{n}-1 / 2}^{1 / 2}(\mu)$ can be represented in terms of elementary functions by explicit evaluation of the integrals given in Hobson [3, (90), page 244 and (91), page 245]:

$$
\begin{aligned}
\left(1-\mu^{2}\right)^{1 / 4} P_{v_{n}-1 / 2}^{1 / 2}(-\mu) & =\sqrt{\frac{\pi}{2}} \cos \left(v_{n} \cos ^{-1}(-\mu)\right), \quad-1<\mu<1, \\
\left(\mu^{2}-1\right)^{1 / 4} Q_{v_{n}-1 / 2}^{1 / 2}(\mu) & =i \sqrt{\frac{2}{\pi}} e^{-v_{n} \cosh ^{-1}(\mu)}, \quad \mu>1 .
\end{aligned}
$$

Thus

$$
W= \begin{cases}-\frac{2}{\pi} \frac{\sin v_{n} \pi}{\pi r r_{0}} \frac{e^{-v_{n} \cosh ^{-1} \mu}}{\left(\mu^{2}-1\right)^{1 / 2}}, & |t|>\left|r+r_{0}\right|, \mu>1 \\ \frac{1}{2 r r_{0}} \frac{\cos \left(v_{n} \cos ^{-1}(-\mu)\right)}{\left(1-\mu^{2}\right)^{1 / 2}}, & \left|r+r_{0}\right|>|t|>\left|r-r_{0}\right| \\ 0, \quad\left|r-r_{0}\right|>|t|\end{cases}
$$


Hence, substituting (2.11) into (2.8) gives

$$
\begin{aligned}
I_{n}= & \frac{1}{r r_{0}} \int_{\left|r-r_{0}\right|}^{\left|r+r_{0}\right|} \frac{\cos \left(v_{n} \cos ^{-1} \mu\right) t e^{i k \sqrt{\left(t^{2}+\left|z-z_{0}\right|^{2}\right)}} d t}{\left(1-\mu^{2}\right)^{1 / 2} \sqrt{\left(t^{2}+\left|z-z_{0}\right|^{2}\right)}} \\
& -\frac{4 \sin v_{n} \pi}{\pi^{2} r r_{0}} \int_{\left|r+r_{0}\right|}^{\infty} \frac{e^{-v_{n} \cosh ^{-1} \mu}}{\left(\mu^{2}-1\right)^{1 / 2}} \frac{e^{i k \sqrt{\left(t^{2}+\left|z-z_{0}\right|^{2}\right)}}}{\sqrt{\left(t^{2}+\left|z-z_{0}\right|^{2}\right)}} t d t .
\end{aligned}
$$

Thus we have reduced the evaluation of (2.3) to that of (2.12) which can be seen to be more suitable for rapid numerical computation since it does not involve Bessel functions.

\section{Free-space Green's function}

It is well known from Duffy [2, (5.1.67), page 285] that

$$
\frac{e^{i k R}}{R}=\sum_{n=-\infty} g_{n}\left(r, z ; r_{0}, z_{0}\right) \cos n\left(\theta-\theta_{0}\right),
$$

where $g_{n}\left(r, z ; r_{0}, z_{0}\right)=\int_{0}^{\infty} h J_{n}(r h) J_{n}\left(r_{0} h\right) e^{-\left|z-z_{0}\right| \sqrt{\left(h^{2}-k^{2}\right)}}\left(d h / \sqrt{\left(h^{2}-k^{2}\right)}\right.$. By using Lambs expression (2.7) with the root determination (2.4), we get

$$
g_{n}\left(r, z ; r_{0}, z_{0}\right)=\int_{0}^{\infty} \frac{t e^{i k \sqrt{\left(t^{2}+\left|z-z_{0}\right|^{2}\right)}}}{\sqrt{\left(t^{2}+\left|z-z_{0}\right|\right)^{2}}}\left(\int_{0}^{\infty} h J_{n}(r h) J_{n}\left(r_{0} h\right) J_{0}(h t) d h\right) d t .
$$

Now by using (2.9), and remembering that $n$ is an integer, we get

$$
\begin{aligned}
g_{n}\left(r, z ; r_{0}, z_{0}\right) & =\frac{1}{2 \pi r r_{0}} \int_{\left|r-r_{0}\right|}^{\left|r+r_{0}\right|} \frac{e^{i k \sqrt{\left(t^{2}-\left|z-z_{0}\right|^{2}\right)}}}{\sqrt{\left(t^{2}+\left|z-z_{0}\right|\right)^{2}}} \frac{P_{n-1 / 2}^{1 / 2}(-\mu)}{\left(1-\mu^{2}\right)^{1 / 4}} t d t \\
& =\frac{1}{2 r r_{0}} \int_{\left|r-r_{0}\right|}^{\left|r+r_{0}\right|} \frac{e^{i k \sqrt{\left(t^{2}-\left|z-z_{0}\right|^{2}\right)}}}{\sqrt{\left(t^{2}+\left|z-z_{0}\right|^{2}\right)}} \frac{\cos \left(n \cos ^{-1}(-\mu)\right)}{\left(1-\mu^{2}\right)^{1 / 2}} t d t .
\end{aligned}
$$

So we have the free-space Green's function representation

$$
\frac{e^{i k R}}{R}=\frac{1}{2 r r_{0}} \sum_{n=-\infty}^{\infty} \cos n\left(\theta-\theta_{0}\right)\left\{\int_{\left|r-r_{0}\right|}^{\left|r+r_{0}\right|} \frac{e^{i k \sqrt{\left(t^{2}-\left|z-z_{0}\right|^{2}\right)}}}{\sqrt{\left(t^{2}+\left|z-z_{0}\right|^{2}\right)}} \frac{\cos ^{-1}(n \cos \mu)}{\left(1-\mu^{2}\right)^{1 / 2}} t d t\right\},
$$

which to my knowledge is a new representation.

Finally, in conclusion, we have derived new representations for the harmonic Green's function for a wedge with Dirichlet or Neumann boundary conditions on its faces. We have also derived a new expression for the three-dimensional free-space Green's function. It is surprising that neither Lamb nor Macdonald discovered these results since the basis of the results exists in the two papers by Lamb [4] and Macdonald [6] that were published consecutively in the same journal. 


\section{References}

[1] J. J. Bowman, T. B. A. Senior, and P. L. E. Uslenghi, Electromagnetic and Acoustic Scattering by Simple Shapes, North Holland Publishing, Amsterdam, 1969, Chapter 6.

[2] D. G. Duffy, Green's Functions with Applications, Studies in Advanced Mathematics, Chapman \& Hall/CRC, Florida, 2001.

[3] E. W. Hobson, The Theory of Spherical and Ellipsoidal Harmonics, Cambridge University Press, Cambridge, 1931.

[4] H. Lamb, On the theory of waves propagated vertically in the atmosphere, Proc. London Math. Soc. (2) 7 (1909), 122-141.

[5] H. M. Macdonald, Electric Waves, Cambridge University Press, Cambridge, 1902.

[6] Note on the evaluation of a certain integral containing Bessel's functions, Proc. Lon. Math. Soc. (2) 7 (1909), 142-149.

[7] H. Poincaré, Sur la polarisation par diffraction, Acta Math. 16 (1892), 297-339 (French).

A. D. Rawlins: Department of Mathematical Sciences, Brunel University, Uxbridge, Middlesex UB8 3PH, UK

E-mail address: anthony.rawlins@brunel.ac.uk 


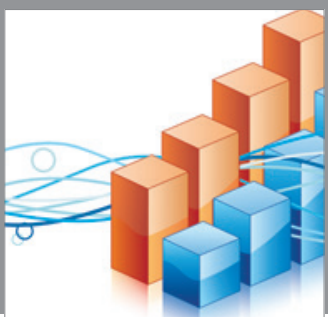

Advances in

Operations Research

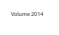

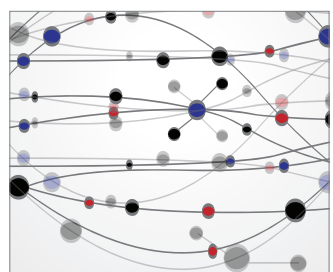

\section{The Scientific} World Journal
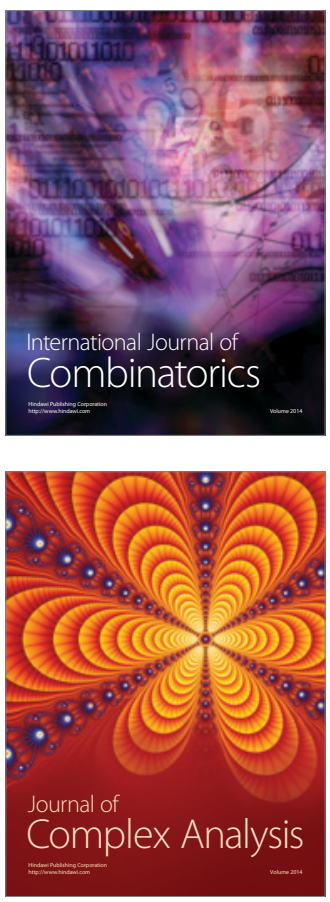

International Journal of

Mathematics and

Mathematical

Sciences
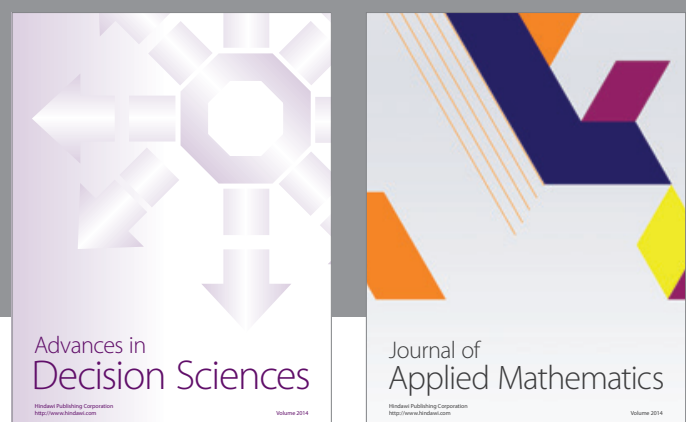

Journal of

Applied Mathematics
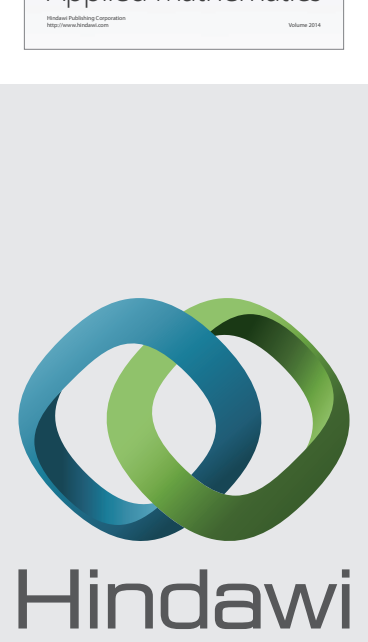

Submit your manuscripts at http://www.hindawi.com
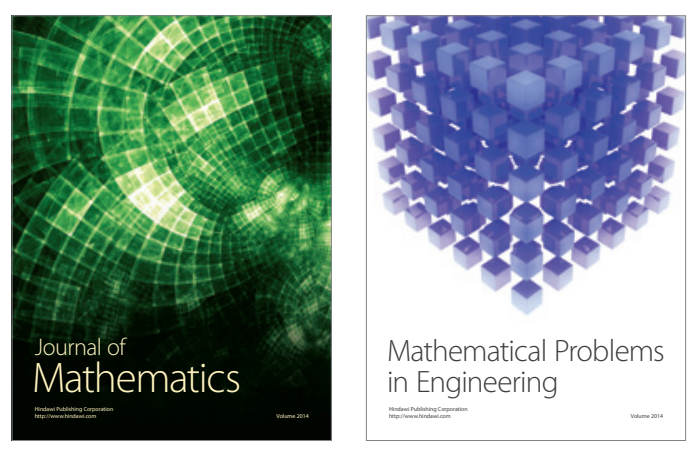

Mathematical Problems in Engineering
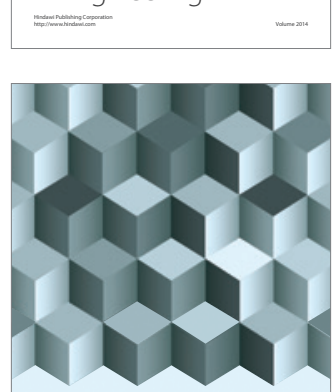

Journal of

Function Spaces
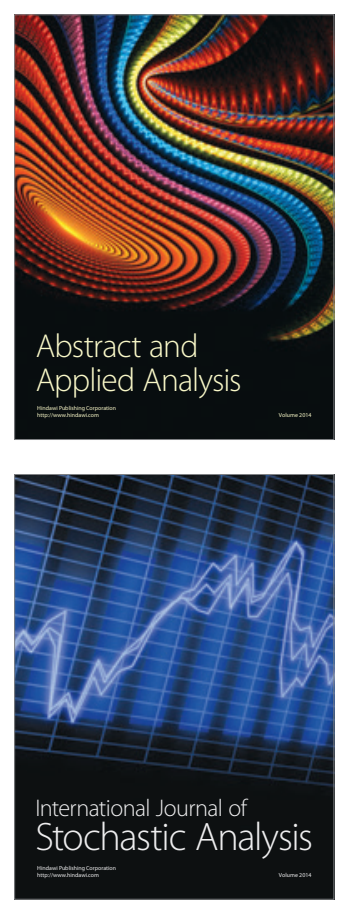

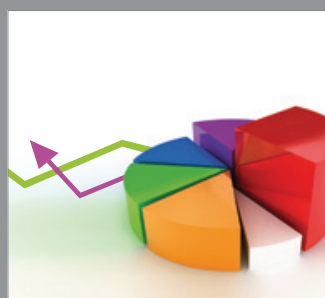

ournal of

Probability and Statistics

Promensencen
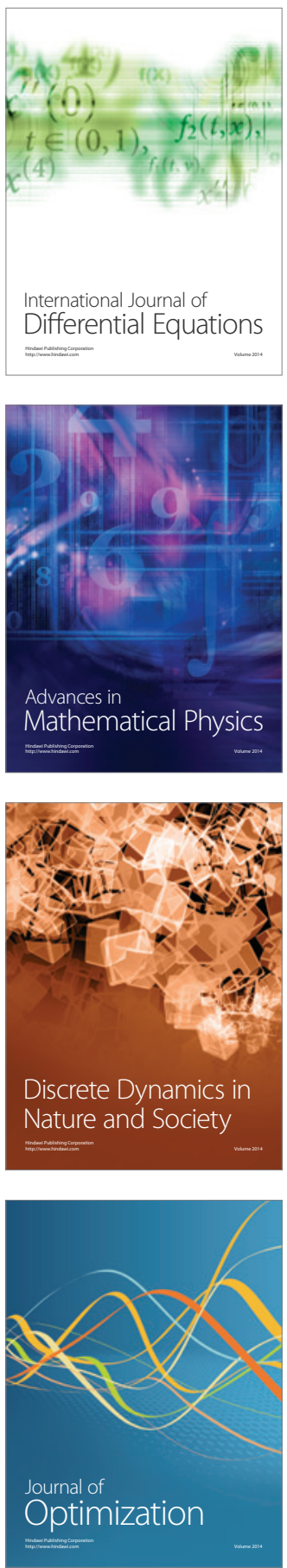